\title{
MODELAGEM DO INCREMENTO DIAMÉTRICO DE Pinus taeda EM FUNÇÃO DE VARIÁVEIS DA COPA E ÍNDICES DE COMPETIÇÃO
}

\author{
Anna Paula Lora Zimmermann ${ }^{1 *}$, Emanuel Arnoni $\operatorname{Costa}^{2}$, Thomas Schröder $^{2}$, Frederico Dimas Fleig ${ }^{2}$ \\ ${ }^{1 *}$ Universidade Federal de Santa Maria, Programa de Pós-Graduação em Engenharia Florestal, Santa Maria, RS, Brasil - \\ zimmermann-a@hotmail.com \\ ${ }^{2}$ Universidade Federal de Santa Maria, Curso de Engenharia Florestal, Santa Maria, RS, Brasil - emanuelarnonicost @hotmail.com; \\ thomaschroder@gmail.com; dimasfleig@uol.com.br \\ Recebido para publicação: 05/08/2015 - Aceito para publicação: 29/11/2015
}

\begin{abstract}
Resumo
Objetivou-se gerar um modelo de incremento periódico em área basal (IPAG) para Pinus taeda válido para povoamentos localizados no Rio Grande do Sul e Paraná. Foram instaladas parcelas temporárias em povoamentos de Cambará do Sul (RS) e Ponta Grossa (PR), em que a árvore de maior diâmetro foi considerada dominante na parcela. De todas as árvores, foi medido o diâmetro à altura do peito e altura total, e das dominantes foi medido também o diâmetro de copa, altura de inserção da copa e comprimento de copa, para o cálculo do grau de esbeltez, proporção de copa, formal de copa, índice de abran gência e saliência. Foi retirado um rolo de incremento de todas as árvores dominantes, para o cálculo do IPAG dos últimos cinco anos. O modelo foi construído pelo método stepwise, no pacote estatístico SAS® e validado pelas condicionantes da regressão. Foram selecionadas as variáveis diâmetro inicial, diâmetro de copa, grau de esbeltez e índice de competição baseado na área basal total e relativa. $\mathrm{O}$ modelo apresentou alto coeficiente de determinação e baixo erro padrão da estimativa, e a análise estatística não demonstrou diferença entre as curvas reais e estimada, evidenciando o uso do modelo em ambos os locais analisados e em locais semelhantes a eles.

Palavras-chave: Árvore individual; método stepwise; relações morfométricas; incremento periódico anual em área basal.
\end{abstract}

\begin{abstract}
Diametric increment modeling of Pinus taeda in function of the crown variables and competition index. The objective was to generate a periodic annual basal area increment (IPAG) model for Pinus taeda valid for stands located in Rio Grande do Sul and Paraná. Temporary plots were installed in stands of Cambará do Sul and Ponta Grossa, where the tree larger diameter was considered domin ant in the sample. All tree diameters at breast height and total height were measured. The dominant trees was also measured the crown diameter, crown insertion height and crown length to calculate $\mathrm{h} / \mathrm{d}$ ratio, crown proportion, crown formal, coverage ratio and crown/trunk diameter ratio. An increment core was obtained from each dominant tree to calculate the IPAG the last five years. The model was built by stepwise method in the statistical package SAS $®$ and validated by regression constraints. The variables initial diameter, crown diameter, degree of slenderness, and competition index based on the total and relative basal area were selected. The model showed high determination coefficient and low standard error of the estimate. The statistical analy sis showed no difference between actual and estimated curves, showing the developed model may be used in both analyzed sites and similar sites.

Keywords: Individual tree; stepwise method; morphometric relationships; periodic annual basal area increment.
\end{abstract}

\section{INTRODUÇÃO}

$\mathrm{O}$ uso de modelos de crescimento tem sido muito difundido co mo uma ferramenta au xiliar às decisões do manejo florestal, principalmente pela sua capacidade de prever e simular a produção madeireira futura. Entretanto, por mais que exista uma gama de modelos já descritos na literatura, eles são baseados em características médias do povoamento, não sendo capazes de explicar as variações pontuais do povoamento (VANCLAY, 1994).

Por outro lado, os modelos para árvores individuais não modelam a produção em função de características médias do povoamento e apresentam resultados mais acurados, pois consideram a árvore como unidade amostral básica. Eles são mais complexos e estimam a produção total do povoamento, através da hipótese aceita de que o crescimento total do povoamento é dado pela soma individual de cada árvore que o

FLOREST A, Curitiba, PR, v. 46, n. 1, p. 115 - 122, jan. / mar. 2016.

Zimmermann, A. P. L. et al.

ISSN eletrônico 1982-4688

DOI: $10.5380 /$ rf.v46il .42424 
compõe (HASENAUER, 2000). A variável resposta pode ser expressa pelo diâmetro ou por sua transformação para área basal, sendo a escolha definida pela praticidade do valor ou preferências do pesquisador. Contudo, Vanclay (1994) recomenda a utilização de incrementos periódicos em curto período de tempo, para diminuição de variações no resultado.

As características dendrométricas, morfo métricas e de competição são consideradas na construção dos modelos de crescimento para árvores individuais, elucidando de forma mais concreta a dinâmica do povoamento, uma vez que esses fatores exercem influência na produção qualiquantitativa de madeira. Devido à confiabilidade desses modelos, seu uso vem sendo difundido em países da Europa e Estados Unidos. No Brasil já é possível encontrar trabalhos como os de Della-Flora (2001), Adame (2008), Mattos (2007) e Cassot et al. (2011), em que os modelos foram baseados em informações de árvores individuais, bem como características do sítio no qual estavam inseridas.

Em florestas equiâneas, a produtividade total é resultante da qualidade do sítio e das condições de concorrência existentes no povoamento. A densidade é expressa nos modelos de crescimento através de índices de competição, dependentes ou independentes da distância entre indivíduos. Es ses índices são capazes de estimar a área ocupada por cada árvore, be m co mo a área mínima necessária para expressão do seu máximo crescimento. Os índices dependentes da distância são considerados melhores descritores da competição, mas essa vantagem não foi confirmada em trabalhos como de Fox et al. (2007) e Chassot et al. (2011). Assim, a utilização de índices independentes da distância torna-se mais vantajosa ao mensurador, devido ao fato de a localização espacial dos indivíduos não interferir nos resultados e, consequentemente, não haver necessidade de medições das coordenadas a campo.

Diante disso, o objetivo deste trabalho foi desenvolver um modelo capaz de predizer o incremento diamétrico de Pinus taeda baseado nas dimensões da árvore e seu grau de competição, na reg ião Sul do Brasil.

\section{MATERIAIS E MÉTODOS}

O estudo foi conduzido no ano de 2010, em povoamentos de Pinus taeda L., todos com 14 anos de idade, em duas áreas $\left(29^{\circ} 06^{\prime} 00^{\prime \prime} \mathrm{S}\right.$ e $50^{\circ} 11^{\prime} 00^{\prime \prime} \mathrm{O} ; 28^{\circ} 53^{\prime} \mathrm{S}$ e $\left.50^{\circ} 07^{\prime} 00^{\prime \prime} \mathrm{O}\right)$ no município de Cambará do Sul, Rio Grande do Sul (RS), e em u ma terceira área $\left(25^{\circ} 09^{\prime} 09^{\prime \prime} \mathrm{S}\right.$ e $\left.50^{\circ} 05^{\prime} 16^{\prime \prime} \mathrm{O}\right)$ no município de Ponta Grossa, Paraná (PR).

O município de Cambará do Sul está localizado na região fisiográfica dos Campos de Cima da Serra, no extremo nordeste do estado do Rio Grande do Sul. De acordo com a classificação climática de Köppen, nessa região predomina o clima do tipo $\mathrm{Cfb}$, com temperatura média anual de $14,9^{\circ} \mathrm{C}$ e precipitação méd ia anual de aproximadamente $1.800 \mathrm{~mm}$ (MORENO, 1961). Os solos são dos tipos Cambissolo Húmico e Alu mínico, podendo ocorrer as sociações com Neossolos Litólicos (STRECK et al., 2008). Os povoamentos de Cambará do Sul foram implantados no ano de 1996, em espaçamento inicial 3 x 2 m. Foi realizada desrama, com limpeza de $60 \%$ da altura total das árvores, além de desbaste sistemático, com a retirada da sexta linha de plantio e desbastes seletivos aleatórios.

O município de Ponta Grossa localiza-se na região do Segundo Planalto Paranaense e, de acordo com a classificação de Köppen, possui clima do tipo pluvial temperado, denominado Cfb, com temperatura média anual de $18^{\circ} \mathrm{C}$ e precipitação média anual de aproximadamente $1.600 \mathrm{~mm}$ (ALVARES et al., 2013). Devido à presença de formações geológicas sedimentares, os solos são pouco desenvolvidos, sendo frágeis e de baixa fertilidade, arenosos e rasos, podendo-se notar as ações dos processos erosivos. O povoamento de Pinus taeda foi implantado em 1996, em espaçamento 3 × 3 m, com a finalidade de pesquisa entre diferentes tipos de clones com a realização somente de desbastes seletivos.

Nos três locais foi utilizado o mesmo método de coleta de dados, com unidades amostrais aleatórias temporárias de forma retangular ou quadrada, de acordo com o espaçamento inicial de plantio. Em cada ponto amostral, considerou-se como centro da parcela e árvore do minante aquela que possuía maior diâmetro à altura do peito em relação às suas vizinhas mais próximas na linha e entrelinha, consideradas dominadas e competidoras. Visto que os povoamentos já haviam recebido desbastes, o número de árvores competidoras em cada unidade a mostral foi variável, podendo ser de no máximo oito árvores (Figura 1). To mou-se cuidado para que as árvores dominantes estivessem afastadas da linha central do desbaste e para que as dominadas não estivessem livres de competição em um dos lados da copa. 


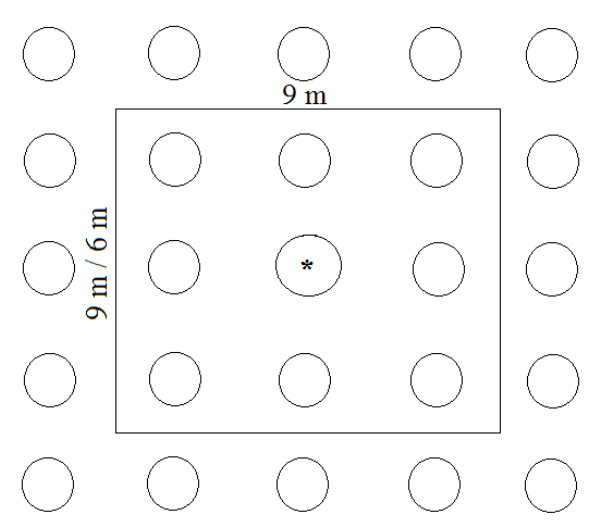

Figura 1. Esquema da instalação das unidades amostrais e medição da árvore dominante (*) e suas competidoras.

Figure 1. Scheme of installation of the sampling units and measurement of the dominant tree (*) and its competitors.

De todas as árvores pertencentes à unidade amostral, foram mensuradas as variáveis diâmetro à altura do peito (DAP), com fita métrica, e altura total (h), com hipsômetro Vertex III. Das árvores dominantes, foram tomadas também as variáveis morfo métricas da copa, como: diâmetro de copa ( $\mathrm{dc})$, dado pela méd ia aritmética de quatro raios de copa; altura de inserção da copa (hi); e comprimento de copa (cc), dado pela diferença entre a altura total e a altura de inserção. As relações morfo métricas calculadas foram: grau de esbeltez $(\mathrm{Ge})=\mathrm{h} / \mathrm{dap}$; proporção de copa $(\mathrm{Pc})=\mathrm{cc} / \mathrm{h} * 100$; formal de copa $(\mathrm{Fc})=\mathrm{dc} / \mathrm{cc}$; índice de abrangência $(\mathrm{IA})=\mathrm{dc} / \mathrm{h}$; e índice de saliência (IS) $=$ dc/dap.

Além disso, foi retirado um rolo de incremento das árvores dominantes com um trado de Pressler, os quais foram medidos com au xílio do sistema Lintab ${ }^{\mathrm{TM}}$ (Linear table) - TSAPTM (Time Series Analysis Program) com precisão de 1/100 mm. O incremento periódico anual em diâmetro foi calculado pela fórmu la, em que: $\mathrm{P}=$ intervalo de medição, no caso, 5 anos

Os índices de competição calculados para os povoamentos foram:

Área basal pontual por hectare (IC1):

$$
I C 1=\left(g_{i}+\sum_{j}^{n} g_{j}\right) * 10000 / A
$$

em que: $\mathrm{g}_{\mathrm{i}}=$ área basal da árvore dominante; $\mathrm{g}_{\mathrm{j}}=$ área basal de cada árvore competidora; $\mathrm{A}$ = área ocupada pela unidade amostral, dependendo do espaçamento de plantio: $3 \times 3 \mathrm{~m}=81 \mathrm{~m}^{2}$ ou $3 \times 2 \mathrm{~m}=54 \mathrm{~m}^{2}$.

Índice de Glover-Holl baseado na altura (IC2):

$$
I C 2=\frac{h_{i}}{\sum_{j}^{n} \bar{h}_{j}}
$$

em que: $h_{i}=$ altura total da árvore dominante; $h_{j}=$ altura total de cada árvore competidora.

Índice de Glover-Holl baseado na altura e no diâmetro (IC3):

$$
I C 3=\frac{d_{i}^{2} \cdot h_{i}}{\overline{d_{\jmath}^{2} \cdot h_{\jmath}}}
$$

em que: $d_{i}=$ diâmetro da árvore dominante; $h_{i}=$ altura total da árvore dominante; $d_{j}$ diâmetro da árvore competidora; $\mathrm{h}_{\mathrm{j}}=$ altura total $\mathrm{da}$ árvore competidora.

Área basal relativa (IC4):

$$
G r=\frac{G}{g i}
$$

em que: $\mathrm{G}$ = área basal da parcela ext rapolada para hectare; $\mathrm{g}_{\mathrm{i}}=$ área basal de cada árvore competidora. 
O modelo de crescimento foi construído a partir do procedimento de seleção de variáveis no programa estatístico SAS® (Statistical Analysis System), versão 9.0, no qual o incremento periódico anual em diâmetro dos últimos 5 anos foi testado como variável dependente. Como variáveis independentes foram testadas as variáveis dendrométricas, morfo métricas e os índices de competição.

Foram realizados testes para verificar as suposições do método dos mínimos quadrados: KolmogorovSmirnov para normalidade dos erros, teste qui-quadrado para homogeneidade de variância e método de DurbinWatson para a independência dos resíduos. A existência de colinearidade foi testada pelo fator de inflação de variância (VIF) de cada coeficiente, que indica que correções devem ser feitas ao modelo quando seu valor for maior do que 15 (CHATTERJEE; HADI, 2006).

\section{RES ULTADOS E DIS CUSSÃO}

As características dendrométricas, morfométricas e de competição das árvores dominantes dos três povoamentos de Pinus taeda analisados e sua estatística básica são apresentadas na tabela 1. Foram mensuradas 20 árvores dominantes em cada local em Cambará do Sul e 25 em Ponta Grossa. No geral, o diâmetro à altura do peito atual e a altura total das árvores apresentaram pouca variação, demonstrando considerável homogeneidade de dimensões entre os indivíduos e povoamentos analisados.

Quanto às características morfométricas, o grau de esbeltez apresentou menor variação, com valor médio de 0,77 . Valores menores que um, como neste caso, são desejáveis para esse índice, significando maior incremento em diâmetro do que altura, e consequentemente maior estabilidade às árvores (SELLE; VUADEN, 2010). Entre todas as variáveis analisadas, os índices de competição calculados apresentaram as maiores variações, evidenciando diferentes graus de competição exercidos pelas árvores vizinhas em relação às árvores dominantes, mas com suas características não consideradas neste estudo.

Tabela 1. Resumo estatístico das variáveis testadas pelo método stepwise para a modelagem do incremento periódico anual em diâmetro (IPAd) de Pinus taeda.

Table 1. Statistical summary of the variables tested by stepwise method for modeling the periodic annual incre ment in diameter (IPAd) of Pinus taeda.

\begin{tabular}{lcccccc}
\hline Características & Variáveis & Min & Méd & Máx & S & CV\% \\
\hline \multirow{3}{*}{ Dendrométricas } & $\mathrm{DAP}$ & 25,1 & 31,6 & 39,2 & 2,8 & 8,9 \\
\cline { 2 - 7 } & $\mathrm{DAP}_{-5 t}$ & 13,2 & 22,5 & 30,9 & 3,9 & 17,3 \\
\cline { 2 - 7 } & $\mathrm{h}$ & 14,1 & 17,2 & 21,0 & 2 & 11,6 \\
\hline \multirow{4}{*}{ Morfométricas } & $\mathrm{dc}$ & 2,8 & 4,1 & 6,6 & 0,7 & 17,1 \\
\cline { 2 - 7 } & $\mathrm{cc}$ & 3,9 & 5,9 & 8,5 & 1,0 & 17 \\
\cline { 2 - 7 } & $\mathrm{Ge}$ & 0,57 & 0,77 & 1,13 & 0,11 & 14,3 \\
\cline { 2 - 7 } & $\mathrm{Pc}$ & 20,6 & 34,7 & 54,5 & 7,0 & 20,2 \\
\cline { 2 - 7 } & $\mathrm{Fc}$ & 0,47 & 0,71 & 1,43 & 0,18 & 25,4 \\
\cline { 2 - 7 } & $\mathrm{IA}$ & 0,16 & 0,24 & 0,35 & 0,04 & 16,7 \\
\hline \multirow{5}{*}{ Competição } & $\mathrm{IS}$ & 0,13 & 0,19 & 0,30 & 0,04 & 21,1 \\
\cline { 2 - 7 } & $\mathrm{IC} 1$ & 33,3 & 56,4 & 76,6 & 9,9 & 17,6 \\
\cline { 2 - 7 } & $\mathrm{IC} 2$ & 0,93 & 1,04 & 1,46 & 0,08 & 7,7 \\
\cline { 2 - 7 } & $\mathrm{IC} 3$ & 1,06 & 1,59 & 2,65 & 0,33 & 20,8 \\
\hline & $\mathrm{IC} 4$ & 258,4 & 577,5 & 1007,5 & 177,7 & 30,8 \\
\hline
\end{tabular}

Mín = mínimo; Méd = média; Máx = máxima; $\mathrm{S}=$ desvio padrão; $\mathrm{CV} \%$ = coeficiente de variação; $\mathrm{DAP}=$ diâmetro à altura do peito atual; $\mathrm{DAP}_{-5 \mathrm{t}}=$ diâmetro in icial; $\mathrm{h}=$ altura total, em metros; dc = diâmetro da copa, em metros; $\mathrm{cc}=$ comprimento da copa, em metros; Ge = grau de esbeltez; Pc = proporção da copa, em \%; $\mathrm{Fc}=$ formal de copa; IA = índice de abrangência; IS = índice de saliência; IC1, IC2, IC3 e IC4 = índices de competição $1,2,3$ e 4 , respectivamente.

A variável dependente a ser explicada neste trabalho foi o incremento periódico anual em diâmetro. Testadas as variáveis dendrométricas, morfométricas e de competição, a que melhor explica o crescimento da espécie, sendo primeiramente selecionada, foi o diâmetro inicial do período de incremento considerado, elucidando cerca de $47 \%$ da variação dos dados. As demais variáveis incluídas foram grau de esbeltez (h/d), diâmetro da copa, índice de competição 1 baseado na área basal pontual por hectare e índice de competição 4 , que leva em consideração a área basal relativa. 
De acordo com o modelo selecionado (Tabela 2), nota-se que o incre mento periódico anual em diâmetro tem u ma relação inversa ao diâmetro apresentado pela árvore no início do período analisado, sendo que as árvores de menor diâmetro inicial foram aquelas que mais aumentaram seu diâmetro no período analisado. Povoamentos jovens, como é o caso dos analisados neste trabalho, ainda possuem espaço de crescimento (OLIVER; LA RSON, 1996) suficiente para que as árvores cresçam sem algum fator limitante.

O diâmetro da copa, também selecionado para compor o modelo, tem uma relação direta com o incremento em diâmetro. A corre lação dess as duas variáveis, também conhecida como Índice de Saliência, é de suma importância ao manejo florestal, sendo capaz de explicar até a qualidade do sítio, uma vez que, quanto menor for essa relação, melhor é a qualidade do sítio (SCHNEIDER, 2008). O índice de saliência calculado neste trabalho está abaixo do encontrado em outras pesquis as com espécies florestais, como Wink et al. (2012), Tonini e Arco Verde (2005) e Hernández e Luna (2008), demonstrando que o potencial de produção dos povoamentos analisados está sendo capaz de ser express o no crescimento das árvores.

As variáveis de competição ta mbém inclusas no modelo levam em consideração a área basal pontual e a área basal relativa, em hectares. A área basal pontual e o incremento em diâmetro possuem uma relação direta, uma vez que é dada pela soma das áreas basais das árvores que compõem a amostra. Assim, quanto maior for o incremento, maior será o diâmetro final e, consequentemente, maior área bas al a a mostra possuirá.

A máxima densidade de ocupação suportada por um sítio depende das dimensões das árvores do povoamento e da densidade. Através da área basal total por hectare $\left(\mathrm{G} / \mathrm{m}^{2}\right)$ de u movoamento é possível fazer inferências sobre o grau de competição estabelecido. Entretanto, essa variável não indica se esse valor é dado pela soma das áreas basais de muitas árvores pequenas ou de poucas árvores grandes. O número de árvores por hectare, proposta por Spurr (1952), ainda é uma das maneiras mais eficazes para expressar a densidade de uma população. Assim, a dimensão das árvores e a área basal relativa possuem u ma relação inversa, uma vez que, conforme as árvores vão aumentando suas dimensões, de maior espaço de crescimento elas vão necessitando, diminu indo o número de indivíduos a ser mantido na área.

Tabela 2. Parâmetros estimados e estatísticas de ajuste e precisão do modelo de incremento periódico anual em diâmetro (IPAd) para Pinus taeda.

Table 2. Estimated parameters and fit statistics and accuracy of the annual periodic increment in diameter model (IPAd) for Pinus taeda.

\begin{tabular}{lcccc}
\hline Variável & Parâmetros estimados & Erro padrão & Pr $>|\mathbf{t}|$ & VIF \\
\hline Intercepto & 3,54340 & 0,31919 & $<0,0001$ & 0,0 \\
DAP $_{-5 t}$ & $-0,14740$ & 0,00709 & $<0,0001$ & 4,5 \\
Dc & 0,80198 & 0,02079 & 0,0002 & 1,1 \\
Ge & 1,16621 & 0,18577 & $<0,0001$ & 2,7 \\
IC1 & 0,04118 & 0,00199 & $<0,0001$ & 2,3 \\
IC4 & $-0,00341$ & 0,00015 & $<0,0001$ & 4,2 \\
\hline $\mathrm{R}^{2}$ & 0,9575 & & & \\
Sy x\% & 5,72 & & & \\
\hline
\end{tabular}

DAP $_{-5 t}=$ diâmetro inicial; dc = diâmetro da copa, em metros; Ge = grau de esbeltez; IC1 e IC4 = índices de competição 1 e 4 , respectivamente; VIF = valor de influência; $\mathrm{R}^{2}=$ coeficiente de determinação; Sy $\mathrm{x} \%=$ erro padrão da estimativa, em $\%$.

Ainda sobre a tabela 2 , a inclusão das cinco variáveis resultou em um modelo com coeficiente de determinação ajustado alto, demonstrando que as variáveis selecionadas foram capazes de explicar 95,75\% da variação total do incremento periódico anual em diâmetro. O erro padrão da estimativa demonstrou que os dados variaram até 5,72\% ao redor da linha média de regressão (Tabela 2). Nenhum dos coeficientes apresentou valor de inflação de variância maior que 15 , demonstrando inexistência de colinearidade e sem necessidade de correções no modelo (CHATTERJEE; HADI, 2006).

A boa performance estatística do modelo é superior quando comparada a outros trabalhos que buscaram modelar o crescimento de povoamentos monoculturais, tais como Padoin (2007), Bueno e Bevilacqua (2010) e Souza et al. (2014). A análise gráfica (Figura 3a) mostra que os valores estimados são muito próximos aos valores reais de incremento. Observa-se também que houve um decréscimo na taxa de incremento conforme a dimensão inicial da árvore, como já comentado anteriormente, devido provavelmente à es tagnação do cres cimento delas.

A dispersão dos resíduos (Figura 2b) também comprova a eficiência do modelo em estimar o incremento periódico anual em diâmetro. Nota-se que não há tendência dos resíduos ao longo da linha média para nenhum dos três locais analisados. Assim, o modelo pode ser utilizado em qualquer um dos locais de estudo, sem tendências quanto às dimensões das árvores analisadas .

FLOREST A, Curitiba, PR, v. 46, n. 1, p. 115 - 122, jan. / mar. 2016

Zimmermann, A. P. L. et al.

ISSN eletrônico 1982-4688

DOI: $10.5380 /$ rf.v46il .42424 

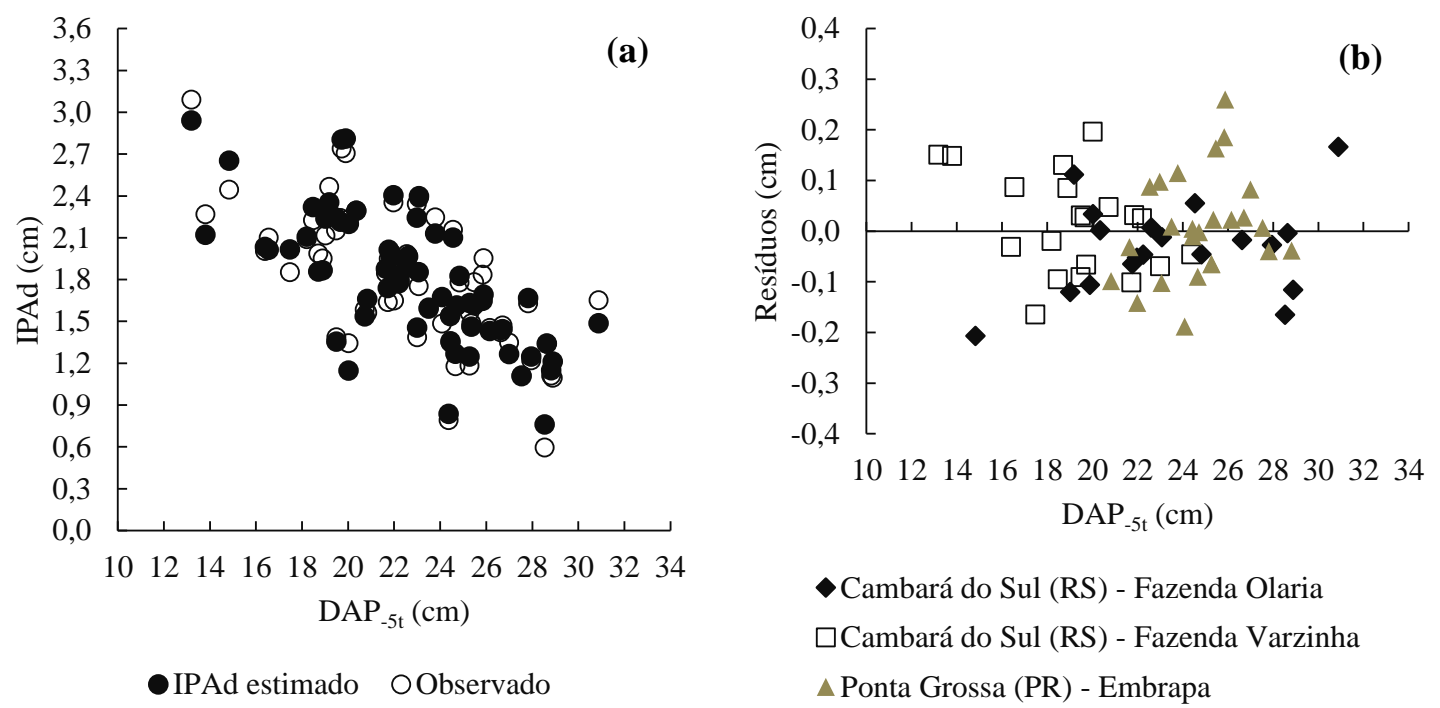

Figura 2. Performance do modelo para o modelo de incremento periódico anual em diâmetro (IPAd) para Pinus taeda: (a) valores de IPAd observados e estimados em relação ao DAP -5 ; (b) distribuição gráfica dos resíduos em relação ao $\mathrm{DAP}_{-5 t}$ para os três locais estudados.

Figure 2. Performance of the model for annual periodic increment in diameter (IPAd) for Pinus taeda: (a) observed and estimated values IPAd in relation to the DAP $-5 t$; (b) graphic distribution of the waste from the $\mathrm{DAP}_{-5 t}$ for the three sites studied.

O teste de aderência de Kolmogorov-Smirnov não foi significativo (Tabela 3), demonstrando que os resíduos seguem uma distribuição normal. Schneider (2009) diz que geralmente variáveis contínuas, como volume, área basal e diâmetro, tendem a se distribuir de forma normal, ao contrário de variáveis de contagem, como nú mero de sementes ou frutos. O teste qui-quadrado, realizado para comparar os valores estimados comos reais, não foi significativo, de monstrando não haver diferenças entre as curvas de incremento real e estimada.

Os resíduos não apresentam correlação em série, u ma vez que o teste de Durbin-Watson não foi significativo, demonstrando que não há dependência entre os resíduos. Assim, ratifica-se a hipótese da possibilidade de o modelo gerado ser utilizado para predição do crescimento da espécie nos estados do Sul do país.

Tabela 3. Testes de condicionantes de regressão do modelo de incremento periódico anual em diâmetro (IPAd) para Pinus taeda.

Table 3. Regression testing conditions of the annual periodic increment model in diameter (IPAd) for Pinus taeda.

\begin{tabular}{|c|c|c|}
\hline \multicolumn{3}{|c|}{ Normalidade dos resíduos } \\
\hline Kolmogorov- & & $\mathrm{Pr}>\mathrm{D}$ \\
\hline 0,09 & & $>0,150$ \\
\hline \multicolumn{3}{|c|}{ Homocedasticidade dos resíduos } \\
\hline Graus de liberdade & $\overline{\chi^{2}}$ & $\operatorname{Pr}>\chi^{2}$ \\
\hline 20 & 22,61 & 0,3084 \\
\hline \multicolumn{3}{|c|}{ Independência dos resíduos } \\
\hline Durbin-Watson D & $\mathrm{Pr}<\mathrm{DW}$ & Pr $>$ DW \\
\hline 2,367 & 0,9061 & 0,0939 \\
\hline
\end{tabular}

\section{CONCLUS ÕES}

- O modelo selecionado pelo método stepwise que melhor explica o incremento em área basal de Pinus taeda em povoamentos da região Sul do país é IPAd $=3,5434-0,1474$. DAP $-5 t+0,80198$.dc +1 1,16621.Ge + 0,04118.IC1 - 0,00341.IC4.

- O modelo gerado apresentou alto coeficiente de determinação e baixo erro padrão da estimativa, 
demonstrando que ele é capaz de explicar a variação em incremento e diâmetro nos locais analisados.

- As variáveis diâmetro inicial, diâmetro da copa, grau de esbeltez e índices de competição baseados na área basal pontual e relativa foram selecionadas pelo método stepwise, evidenciando sua influência na taxa de incre mento apresentada pelas árvores analisadas.

- De acordo com os testes estatísticos realizados, o modelo gerado pode ser utilizado para predição do incremento periódico em área basal de Pinus taeda em locais semelhantes aos povoamentos analisados neste trabalho.

\section{REFERÊNCIAS}

ADAME, P.; DEL-RÍO, M.; CAÑELLAS, I. A mixed nonlinear height-diameter model for pyrenean oak (Quercus pyrenaica Willd.). Forest Ecology and Management, A msterdam, v. 256, n. 1/2, p. 88 - $98,2008$.

ALVARES, C. A.; STAPE, J. L.; SENTElHAS, P. C.; MORAES, G.; LEONARDO, J.; SPAROVEK, G. Köppen's climate classification map for Brazil. Meteorologische Zeitschrift, v. 22, n. 6, p. 711 - 728, 2013.

BUENO, S.; BEVILACQUA, E. Modeling stem increment in individual Pinus occidentalis Sw. trees in La Sierra, Dominican Republic. Forest Systems, v. 19, n. 2, p. 170 - 183, 2010.

CHASSOT, T.; FLEIG, F. D.; FINGER, C. A. G.; LONGHI, S. J. Modelos de crescimento em diâmetro de árvores individuais de Araucaria angustifolia (Bertol.) Kuntze na Floresta Ombrófila Mista. Ciência Florestal, Santa Maria, v. 21, p. 303 - 313, 2011.

CHATTERJEE, S.; HADI, A. S. Regression analysis by example. 2. ed. Hoboken, NJ: John Wiley \& Sons, 2006.

DELlA FlORA, J. B. Modelos de Crescimento para Árvores Singulares - Nectandra megapotamica (Spreng.) Mez. e Ocotea puberula Ness. Dissertação (Mestrado) - Centro de Ciências Rurais, Universidade Federal de Santa Maria, Santa Maria, 2001.

FOX, J. C.; BI, H.; ADES, P. K. Spatial dependence and individual-tree growth models. I. Characterising spatial dependence. Forest Ecology and Management, A msterdam, v. 245, p. 10 - 19, 2007.

HASENA UER, H. Princípios para a modelagem de ecossistemas florestais. Ciência \& Ambiente, Santa Maria, v. 20 , p. $53-69,2000$.

HERNÁ NDEZ, E. H.; LUNA, J. A. N. Relaciones morfométricas de un bosque coetáneo de la región del El Salto, Durango. Ra Ximhai, El Fuerte, v. 4, n. 1, p. 69 - 81, 2008.

MACHADO, S. A.; AGUIAR, L. P.; FIGUEIREDO FILHO, A.; KOEHLER, H. S. Modelagem do volu me do povoamento para Mimosa scabrella Benth. na Região Metropolitana de Curitiba. Revista Árvore, Viçosa, v. 32, n. 3, p. $465-478,2008$.

MATTOS, R. B. Produti vidade e incremento de Cabralea canjerana (Vell.) Mart., Cedrela fissilis Vell. e Cordia trichotoma (Vell.) Arrab. Ex Steud., em floresta nativa no Rio Grande do Sul. Santa Maria, 105 f. Tese (Doutorado em Engenharia Florestal) - Universidade Federal de Santa Maria, 2007.

MORENO, J. A. Clima do Rio Grande do Sul. Porto Alegre: Secretaria da Agricultura, 1961, 41 p.

OLIVER, C. D.; LARSON, B. C. Forest stand dynamics. New York: McGraw-Hill, 1996, 467 p.

PADOIN, V. Diretrizes para desbaste de Pinus taeda L. em função da altura dominante. 95 f. Dissertação (Mestrado em Engenharia Florestal) - Universidade Federal de Santa Maria, Santa Maria, 2007.

SAS INSTITUTE INC. Statistical Analysis System user's guide. Version 9.0. Cary, Statistical Analysis System Institute. 513 p. 2002.

SCHNEIDER, P. R.; SCHNEIDER, P. S. P. Introdução ao manejo florestal. Santa Maria: UFSM. 566 p. 2008.

SELLE, G. L.; VUADEN, E. Crescimento de seis espécies nativas na região central do estado do Rio Grande do Sul. Ambiência, Guarapuava. v. 6, n. 1, p. 169 - 192, 2010.

SOUZA, R. F.; MACHADO, S. A.; FIGUEIREDO FILHO, A. Modelagem da biomassa total e da lenha por unidade de área para bracatingais nativos. Revista Ár vore, Viçosa, v. 38, n. 1, p. 53 - 62, 2014. 
SPURR, S. Forest inventory. New York: The Ronald Press Company, 1952, 476 p.

STRECK, E. V.; KÄMPF, N.; DALMOLIN, R. S. D.; KLAMT, E.; NASCIMENTO, P. C.; SCHNEIDER, P.; GIASSON, E.; PINTO, L. F. S. Solos do Rio Grande do Sul. 2. ed. Porto Alegre, EMATER/RS-ASCA R, 2008. $222 \mathrm{p}$.

TONINI, H.; A RCO-VERDE, M. F. Morfo logia da copa para avaliar o espaço vital de quatro espécies nativas da Ama zônia. Pesquisa Agropecuária Brasileira, Brasília, v. 40, n. 7, p. 633 - 638, 2005.

VANCLAY, J. K. Modelling forest growth and yield. Applications to mixed tropical forests. Wallingford: CA B International, 1994. 312 p.

WINK, C.; MONTEIRO, J. S.; REINERT, D. J.; LIBERALESSO, E. Parâmetros da copa e a sua relação com o diâmetro e altura das árvores de eucalipto em diferentes idades. Scientia Forestalis, Piracicaba, v. 40, n. 93, p. $57-67,2012$. 